\title{
Die professionellen Praktiken in der Berufs- und Weiterbildungsberatung - ein komplexes Innenleben
}

\author{
Wiltrud Gieseke • Maria Stimm
}

Eingegangen: 9. März 2015 / Angenommen: 10. Juli 2015 / Online publiziert: 28. August 2015

(C) Die Autor(en) 2015. Dieser Artikel ist auf Springerlink.com mit Open Access verfügbar.

Zusammenfassung Der Artikel setzt sich grundlagentheoretisch mit der Bedeutung von Entscheidungsprozessen bezogen auf die professionellen Praktiken in der Berufs- und Weiterbildungsberatung auseinander. Über eine empirische Detailanalyse werden Beratungsverläufe dargestellt. Diese Analyse stützt sich auf Beratungsgespräche, die von den Beratenden selbst ausgewählt und zur Verfügung gestellt worden sind. Die Beratungsgespräche spiegeln somit die stattfindende Praxis der Beratung wider. Die Analyseergebnisse im Rahmen einer dem Gegenstand angepassten Dialogmusteranalyse werden an die theoretischen Grundlegungen rückgebunden.

Schlüsselwörter Entscheidungsfindung · Professionelle Praktiken ·

Dialogmusterspezifische und dialogmusterunspezifische Ziele $\cdot$ Berufs- und Weiterbildungsberatung

\section{Professional practices in career counseling and continuing counseling: complex interrelations}

\begin{abstract}
The article deals with the importance of decision-making processes referring to professional practices in career counseling and continuing counseling. Based on an empirical analysis of specific details the process of counseling is being illustrated. Parts of this analysis are the counseling interviews that were selected and
\end{abstract}

Prof. Dr. W. Gieseke · M. Stimm, M.A. $(\bowtie)$

Humboldt-Universität zu Berlin,

Unter den Linden 6, 10099 Berlin, Deutschland

E-Mail: maria.stimm@hu-berlin.de

Prof. Dr. W. Gieseke

E-Mail: wiltrud.gieseke@cms.hu-berlin.de 
provided by the consultant him- or herself. Therefore the counseling interviews represent the common practice of the counseling process. The analysis was conducted as an analysis of dialogue patterns. The results of the analysis are being linked to the theoretical background.

Keywords Decision making · Professional practices · Career counseling · Continuing counseling $\cdot$ Dialog specific aims $\cdot$ Dialog unspecific aims

Inzwischen können wir auf eine sehr breit ausdifferenzierte Beratungspraxis im gesamten pädagogischen Feld blicken, ohne dass aber die Theorieentwicklung und empirische Forschung Schritt gehalten hätten. Die Weiterbildung betreffend ging es in den letzten Jahren vor allem darum, über Förderungen Strukturen für die Weiterbildungsberatung zu schaffen (u. a. Hessencampus - Lebensbegleitendes Lernen (Kruse et al. 2009; Schemmann und Seitter 2013); Referenzmodell für die Koordination bildungsbereichs- und trägerübergreifender Bildungsberatung im Rahmen eines Bildungsmanagements auf kommunaler Ebene (Gieseke et al. 2012); Niedersächsische Modellprojekte (Agentur für Erwachsenen- und Weiterbildung 2013)) und Qualitätskriterien zu entwickeln (Arnold et al. 2009; Schiersmann und Weber 2013; Schröder und Schlögl 2014) sowie diese Aspekte wissenschaftlich zu begleiten. Das heißt, man erhofft sich durch die Entwicklung von regionalen Netzwerkstrukturen, unter anderem durch die Beteiligung vieler Weiterbildungsträger, eine stabile Weiterbildungsberatung, zumindest als Orientierungsberatung (Gieseke 2013). In den Bundesländern wurden hier jeweils bottom-up Strukturen entwickelt. Der Erhalt von Unabhängigkeit sowie Professionalität spielt dabei eine entscheidende Rolle. Die Beratungsprozesse mit den Ratsuchenden sind im Rahmen dieser strukturell-organisationalen Entwicklungen natürlich nicht im Fokus. Hier setzt nun unser Untersuchungsinteresse an: Wir interessieren uns für die Verlaufsformen von Beratung in ihren realisierten Praktiken. Beachtung findet in der allgemeinen Diskussion dabei ein eher betriebswirtschaftliches Regulativ, das auf Zeit und Intensität der Beratung Einfluss nimmt. Dazu gibt es ein „,kritisches Gemurmel“, aber nicht ausreichend empirische Befunde, die den Wert von Kurzzeitberatung und Beratung mit längerer Zeitdauer über Bildungs- und Berufsentscheidungen differenziert einordnen und Folgewirkungen für eine Strukturentwicklung erfassen.

\section{Detailforschung im Rahmen pädagogischer Beratungsforschung}

Wir verstehen unter Detailforschung - in diesem Fall Forschung über Beratung -, diejenige Forschung, die ihre Aufmerksamkeit auf einzelne Aspekte des komplexen Vorganges Beratung lenkt, z. B. auf die Funktion des Wissens oder die Bedeutung von Emotionen in der Beratung. Erst im Zusammenspiel dieser und anderer Aspekte ergibt sich ein Bild über Bildungsberatung. Wir halten pädagogische Forschung als Detailforschung, die sich der Beratungspraxis mit ihren unterschiedlichen institutionellen Bedingungen und verschiedenen Verläufen widmet, ohne die Beratung wer- 
tend zu kommentieren, somit für notwendig, um eine relativ unabhängige Dimension von Professionalität in der Beratung zu erreichen.

In unserem Fall geht es darum, einzelne kommunikative Teilziele, die in der Beratung durchlaufen werden, zu identifizieren. Ethischer Bezugspunkt ist dabei, ob die subjektive Entscheidungsfähigkeit mit Blick auf den eigenen Lebenslauf - im engeren Sinne daher auch Bildungs-, Qualifikations- sowie Kompetenzentscheidungen betreffend - in der Beratung gestärkt wird. Bildungsentscheidungen, wenn sie subjektiv verantwortet werden sollen, und das müssen sie, wenn wir vom selbstverantwortlich handelnden Individuum ausgehen (Wrana 2015), können nicht einem Steuerungsprozess durch Beratung ausgeliefert werden. Dort, wo der subjektive Entscheidungsspielraum eingebunden wird durch beratungsexterne Dritte, entweder als Zuweisung oder als Beurteilung, können wir von regulativer oder gesteuerter Beratung sprechen. Regulative Beratung kann ebenso ordnungspolitisch gemeint sein und die Ermöglichung von Zugängen zur Beratung durch Finanzierung umfassen (Käpplinger et al. 2013). Regulativ ist dann kein Gegenbegriff zur subjektiven Entscheidungsfindung, sondern ermöglicht diese erst für alle sozialen Milieus. Sozialpolitisch begründete Steuerung kann somit nur soweit gehen, als dass sie dem Individuum eine Chance zur Partizipation bietet. Göckler (2012) verweist darauf, dass keine Beratung - auch im Sinne des Fallmanagements - ohne individuelle Spielräume ethisch zu verantworten ist. Für theoretische und empirische Arbeiten ist es daher nicht nur aus ethischen Gründen notwendig, diese Unterscheidung beizubehalten, da sie die potentiellen individuellen Spielräume einschränken kann.

Über Detailforschung ist es möglich, den ganzheitlichen Beratungsprozess empirisch zu zerlegen, um die Theoriebildung in einer interdisziplinären, aber bildungswissenschaftlich ausgerichteten Perspektive voranzubringen. Entsprechend angelegte empirische Untersuchungen, die an der Humboldt-Universität zu Berlin realisiert werden konnten, liegen zur Form und Bedeutung von Fragen in der Beratung (Müller 2005), zur Kategorie Wissen (Enoch 2011) und zur Kategorie Emotionen (Schreyögg 2015) im Beratungsprozess vor. Ebenso können in diesem Zusammenhang Forschungen im Rahmen der Gründungsförderung zu Mischstrukturen in der Entscheidungsfindung und Finanzierungssteuerung (Meier-Gutheil 2009) sowie zur regulativen Beratung über Gutscheine (Käpplinger et al. 2013) angeführt werden.

Die im Folgenden diskutierten Teilbefunde zu Bildungsberatungspraktiken (Gieseke et al. 2014; Gieseke und Stimm 2015, i. B.) gehören zur Detailforschung, die sich in diesem Fall auf die Verlaufsformen von Beratungen konzentriert. Sie geht auf das Zusammenspiel von Teilaspekten des professionellen Handelns in der Beratung ein.

\section{Theoretische Grundannahmen}

Die Theorien zur Beratung haben sich lange - und tun das zum Teil auch heute noch bedingt durch das jeweilig Praxisfeld - auf therapeutische Prämissen ausgerichtet oder sind von der Begründungslogik besonders sozialpädagogisch fokussiert, da Beratung in der Sozialpädagogik die Interventionsstrategie für Veränderungen im Lebenslauf darstellt. Theorien zur Beratung, die auf eine längere Praxis zurückblicken, konnten durch ihre Nähe zum Interesse an einer Entscheidungsfindung - vor 
allem im klientenzentrierten Ansatz - am ehesten für Beratungsprozesse genutzt werden. Der klientenzentrierte Ansatz hatte daher als theoretischer Referenzpunkt eine große Beliebtheit in der Bildungs- und Berufsberatung (Schiersmann und Remmele 2004). Hinzu kamen lösungs- und ressourcenorientierte Beratungskonzepte, die auf eine schnelle Verbesserung von Lebensbedingungen abzielen und datenintensive (berufsbiografisch abschluss- und zertifikatorientierte) Entscheidungsstrukturen fokussieren. Die systemische Beratung hat in der Familientherapie eine hohe Bedeutung, ebenso aber auch in der betrieblichen Weiterbildungsberatung, weil die Abhängigkeit von Entscheidungen hier rückgebunden ist an viele Personen und ihre multiplen Beziehungsverhältnisse (Schiersmann und Thiel 2012).

Jede Detailforschung nutzt theoretische Annahmen und versichert sich bei der inhaltlichen Betrachtung von Beratung interdisziplinär zurück. Bezogen auf die empirische Anlage und den Beratungsprozess sind in unserem Fall theoretische Auseinandersetzungen mit dem Praktiken-Begriff und dem Entscheidungsbegriff grundlegend (Gieseke 2014b; Gieseke und Stimm 2015, i. B.).

\subsection{Entscheidungsfindungen}

Bildungs- und Berufsentscheidungen sind grundlegende Entwicklungsanforderungen in der Moderne, die vom Subjekt, wenn auch in Abhängigkeit von den jeweiligen Milieukonstellationen (Tippelt und Barz 2007), individuell getroffen werden und zu verantworten sind. Entscheidungstheorien sind bisher vor allem für Entscheidungsprozesse in Unternehmen aufgearbeitet worden. Dabei wird hier zwischen deskritptiven und präskriptiven Entscheidungstheorien unterschieden. Deskriptive Theorien untersuchen, wie es zu bestimmten Entscheidungen gekommen ist, präskriptive Theorien stellen die Einflussfaktoren zusammen, unter denen Entscheidungen zu fällen sind. Grundlagentheoretisch wird auf Unternehmen bezogen hierbei von der normativen Annahme ausgegangen, dass alle Individuen als homo oeconomicus agieren. Diese Annahme wird inzwischen in Diskussionen über Entscheidungsprozesse nicht ohne Grund in Frage gestellt.

In den ersten Berufsberatungstheorien und -konzepten wurden besonders präskriptive Theorien genutzt. Es wurde jedoch auch erkannt, dass Fragen des Interesses auf der Grundlage emotionaler Dispositionen einen hohen Einfluss haben (Ertelt und Schulz 2002). Unsere Auswertungen der Emotionsforschung und neurobiologischen Forschung, auch unter Hinzuziehung systemischen Denkens, machen es notwendig, Entscheidungsprozesse in ihren komplexen Abhängigkeiten inter- und intrapsychisch $\mathrm{zu}$ verstehen. Das gilt ganz besonders dann, wenn es um Lebens- und Bildungsentscheidungen geht. Grundlagentheoretisch kann man festhalten: Bei der Entscheidung handelt es sich nicht um ,,isolierte Willensakte“, sondern in der Regel um ,einen zeitlich mehr oder weniger ausgedehnten, ,reifenden' Prozess, in den Überzeugungen, Erwartungen, Erfahrungen, Grundhaltungen mit eingehen, also letztlich die persönliche Lebensgeschichte des Handelnden“" (Fuchs 2008, S. 329).

Viele - besonders in den Bildungswissenschaften - haben Sorge, dass die verstärkte Beachtung der bildungsemotionalen Komponente die rationalen Aspekte in Frage stellt und sogenannte „Bauchentscheidungen“ für komplexen Entscheidungen als theoretische Grundlage angenommen werden (Gigerenzer 2007). Neurobiologi- 
Abb. 1 Lange Wege der Entscheidung (Gieseke 2014a, S. 12)

\begin{tabular}{|c|c|c|c|c|c|}
\hline 1. Phase & 2. Phase & \multicolumn{2}{|c|}{$\begin{array}{l}\text { 3. Phase } \\
\text { (Beratungssituation selbst) }\end{array}$} & 4. Phase & 5. Phase \\
\hline $\begin{array}{l}\text { Überle- } \\
\text { gung eine } \\
\text { Beratung } \\
\text { aufzu- } \\
\text { suchen }\end{array}$ & \begin{tabular}{|l} 
eine Bera- \\
tung auf- \\
suchen \\
oder \\
durch \\
allgemei- \\
ne Orien- \\
tierungs- \\
beratung \\
an eine \\
spezifi- \\
sche Bera- \\
tung ver- \\
wiesen \\
werden
\end{tabular} & \begin{tabular}{|l} 
a) Biografisch \\
Beratungs- \\
atmosphäre, \\
Beratungs- \\
praktiken
\end{tabular} & $\begin{array}{l}\text { le Grundlegung } \\
\text { stauslegung, } \\
\text { nale, Kompetenzen) }\end{array}$ & \begin{tabular}{|l} 
Selbstre- \\
flexion, \\
Gespräche \\
mit Freun- \\
d/inn/en \\
und \\
Familie, \\
professio- \\
neller Aus- \\
tausch \\
unter \\
Bera- \\
tenden
\end{tabular} & $\begin{array}{l}\text { Entschei- } \\
\text { dungsfin- } \\
\text { dung } \\
\text { des/der } \\
\text { Ratsu- } \\
\text { chenden, } \\
\text { Rück- } \\
\text { kopplung } \\
\text { und Frage } \\
\text { nach der } \\
\text { Wirksam- } \\
\text { keit }\end{array}$ \\
\hline
\end{tabular}

sche Befunde und darauf aufbauend philosophische Überlegungen belegen nachhaltig, dass Emotionen und Kognitionen bei Entscheidungen zusammenspielen, wobei in den Emotionsmustern die Sozialisationsprozesse eingelassen sind und ihnen deshalb eine große Wirkung beim Treffen von Entscheidungen zukommt. Dieses belegt indirekt auch die Sozialisationsforschung. Entscheidungen basieren dabei immer auf einer Bearbeitung der Emotionsmuster und sind darauf angewiesen, dass Wissen und kognitive Potentiale abgerufen werden können (in Auswahl Damasio 2000, 2003; Roth 2001, 2008; Fuchs 2010; Perler 2011). Durch die neurobiologische Forschung zeigt sich, dass die Strukturen im Gehirn enthierarchisiert arbeiten. Gleichzeitig wird jedoch sichtbar, wie abhängig der Mensch in seiner Urteils- und Entscheidungsfähigkeit davon ist, rationale Abwägungen leisten zu können und ausdifferenzierte emotionale Fähigkeiten zu nutzen, um Strategien in der Umwelt zu begreifen. Ausdifferenzierungen können Entscheidungen erbringen, die über das Milieu hinausgehen. Letztlich nehmen aber Emotionen den größten Einfluss auf alle Entscheidungsprozesse (Gieseke 2009), deshalb verdienen sie besondere Beachtung.

Für Beratungsprozesse bedeutet das, wenn es um Entscheidungen im Rahmen der zukünftigen Berufs- und Weiterbildungswahl geht, dass es eine Chance gibt, die individuellen Interessen in ihren Ambivalenzen, das vorhandene Wissen über Berufs- und Weiterbildungsstrukturen sowie die bisherigen biografischen Verläufe zu erschließen und aushandeln zu können. Aus dieser Perspektive regt Bildungs- und Berufsberatung an, sich Perspektiven zu erarbeiten und über bisherige Entscheidungen und bisheriges Handeln nachzudenken, aber die Entscheidungen trifft das Individuum nicht in der Beratung selbst. Dies setzt hohe Beratungskompetenz bei den Beratenden voraus. Um den zeitbeanspruchenden Prozess für das Aufsuchen der Beratung, die Beratungssituation an sich und die Entscheidungsfindung nach der Beratung zu verdeutlichen, sind einzelne Phasen in Abb. 1 benannt.

\subsection{Professionelle Praktiken}

Die professionellen Praktiken der konkreten Beratung sind Voraussetzungen dafür, dass die individuelle Entscheidungsfähigkeit vorbereitet und damit ermöglicht wird. Wir sprechen deshalb von professionellen Praktiken, da es sich in der Berufs- und 
Weiterbildungsberatung in der Regel um professionell ausgebildete Beratende handelt, die gleichwohl durch die Dauer ihrer Tätigkeit und durch institutionelle Vorgaben individuelle Muster des Beratens, die als Praktiken wirken, ausgebildet haben. Jede Beratung ist für die Ratsuchenden ein Ausgangspunkt für Entscheidungen. Im Prozess der Entscheidungsfindung setzen sie sich in der Beratung lernend mit Bedingungen und Möglichkeiten auseinander. In diesem Sinne ist Beraten ein spezifisch pädagogisches Handeln und wird zu einer interventionistischen Praktik, die aber in ihrem Anspruch auf die Selbstentscheidung nach der Beratung abzielt.

Lippitt und Lippitt (1999), Fuhr (2003) sowie Thiel (2003) haben Vorschläge für die Strukturierung von Beratungsgesprächsverläufen erarbeitet. Diese Modelle und Konzepte unterstellen, dass es am Ende der Beratung eine Entscheidung gäbe. Das Modell von de Cuvry et al. (2009) macht hier eine Ausnahme und bietet verschiedene kategoriale Durchgänge bei der Betrachtung der Verlaufsprozesse an. Sie erhöhen dadurch die Möglichkeit, die Komplexität des Beratungsprozesses zu erfassen. Ihr theoretischer Ansatz betrachtet den Beratungsverlauf entsprechend nicht nur als Ablaufschema, sondern aus verschiedenen Perspektiven als mehrdimensionales Strukturmodell. Dabei ist auch den anderen Modellen wichtig, dass der Beratungsprozess nicht als linear verlaufender Interaktionsprozess interpretiert wird. Damit zeigen alle, dass es keine eindeutigen Phasenverläufe gibt, sondern eher Wiederholungsschleifen die Beratungsverläufe kennzeichnen.

Bezugnehmend auf Reckwitz' Praxistheorie $(2000,2003)$ gehen wir davon aus, dass es eine Alltagsevidenz des täglichen beraterischen Handelns gibt, die bestimmt ist von den Kontextanforderungen der beratenden Institution, von den professionellen Kompetenzen und inzwischen auch von vorgelagerten Qualitätsansprüchen. In dieser Mischstruktur realisieren sich professionelle Praktiken. Sie folgen selten idealen Ansprüchen, sind nicht nur bestimmt durch den Kontext und ebenso wenig allein erklärbar durch Mentalstrukturen der Beratenden. Sie sind aber auch nicht radikal konstruktivistisch-systemisch zu erklären, da die Individuen selbst dem System nicht in Gänze ausgeliefert sind und gerade die Bildungsfähigkeit des Individuums durch die Nicht-Abgeschlossenheit gegenüber der Umwelt wirksam ist. Es geht um ,ein praktisches Wissen, ein Können, ein know how, ein Konglomerat an Alltagstechniken, ein praktisches Verstehen im Sinne eines, Sich auf etwas verstehen'. Der ,Ort ${ }^{\star}$ des Sozialen ist damit nicht der (kollektive) ,Geist‘ und auch nicht ein Konglomerat von Texten und Symbolen (erst recht nicht ein Konsens von Normen), sondern es sind die ,sozialen Praktiken', verstanden als know-how abhängige und von einem praktischen ,Verstehen' zusammengehaltene Verhaltensroutinen, deren Wissen einerseits in den Körpern der handelnden Subjekte, inkorporiert' ist, die andererseits regelmäßig die Form von routinisierten Beziehungen zwischen Subjekten und von ihnen ,verwendeten“ Artefakten annehmen" (Reckwitz 2003, S. 289).

Für die realisierten Praktiken in den von uns untersuchten Beratungsfällen müssen wir feststellen, dass wir, genau wie im oben beschriebenen Sinne, einen bestimmten Typus von Beratung für die Berufs- und Weiterbildung mit sich wiederholenden thematisch-inhaltlichen sowie beziehungsförderlichen Strukturen vorfinden. 


\section{Anlage der Untersuchung}

Uns standen für die Auswertung 31 Transkripte von durchgeführten Berufs- und Weiterbildungsberatungsgesprächen aus relevanten Institutionen zur Verfügung. Dabei sind die beratenden Institutionen nicht in die Untersuchung miteinbezogen, dies wäre eine andere Forschungsfrage. Die Beratungsgespräche wurden uns nach einer Bitte, sich an diesem Forschungsprojekt zu beteiligen, von den Beratenden mit Akzeptanz der Ratsuchenden zugeschickt. Die Auswahl der Beratungsgespräche erfolgte durch die Beratenden selbst im Sinne von prototypischen Beratungsverläufen für ihr Beratungshandeln. Mit den Beratenden wurden nach Vorlage der Transkripte zusätzlich Interviews geführt, welche sich auf die theoretischen Positionen zur Beratung, allgemeine Einschätzungen von Beratung und auf den jeweiligen im Transkript abgebildeten Beratungsfall konzentrierten. Als methodische Erkenntnis können wir diesbezüglich festhalten, dass Interviews mit Beratenden sehr wenig über den praktischen Beratungsverlauf aussagen, da die Beratenden in ihrer professionellen Außenpräsens einen anderen Fokus setzen. Dieser Aspekt macht jedoch Praktiken aus, sie sind nicht unmittelbar reflexiv verfügbar. Nicht durchgeführt werden konnten aufgrund der fehlenden Finanzierungsbedingungen und des Datenschutzes Interviews mit den Ratsuchenden.

Der Auswertungsvorgang erfolgte in einer vierköpfigen Forscherinnengruppe. Wir gehen davon aus, dass qualitative Forschung dieser Art in der Regel durch mehrere Personen geleistet werden sollte. Im ersten Durchgang fand eine theoretische Auseinandersetzung mit dem Praktiken-Begriff und mit methodischen Ansätzen zur Auswertung statt. Der methodische Zugriff zielt hier nicht auf Typen üblichen sozialen Handelns als ethnografisches Moment (Bohnsack 2008), ebenso wenig auf psychosoziale Dispositionen, die sich im Dialog wiederholen (Deppermann et al. 2010), und auch nicht auf den Ansatz, Beratungsgespräche als allgemeine Alltagsphänomene im Vergleich mit sonstigen Gesprächen, wie im Fall der Konversationsanalyse, zu betrachten. Die Auswahl des methodischen Zugriffs erfolgte letztlich aus bildungswissenschaftlicher Sicht, um die professionelle Struktur von Beratung in den realisierten Praktiken sichtbar zu machen. Für die Analyse der Beratungsgespräche haben wir den Ansatz von Kohl (1986) genutzt. Mit diesem Ansatz, der von Dialogmustern als interpretative Verknüpfung zwischen Äußerungsereignissen und Handlungskonstituenten ausgeht, kann der professionelle Aspekt von Beratung besonders gut im Detail bearbeitet werden. Als primäres Kommunikationsziel, welches nach Kohl (1986) das Dialogmuster entscheidend bestimmt, können wir für unsere Perspektive auf die Beratungsgespräche den Entscheidungsprozess festhalten. Das primäre Kommunikationsziel ist dabei durch verschiedene kommunikative Teilziele geprägt, die im Fall der Beratung die Bedingungen schaffen, entscheidungsfähig zu werden, hier für Bildungs- und Berufsentscheidungen.

Wir haben in einem ersten Materialdurchgang induktiv Kategorien identifiziert und ausdifferenziert. Kategorien beziehen wir in unserem Fall auf die kommunikativen Teilziele nach Kohl (1986), der diese untergliedert in dialogmusterspezifische und dialogmusterunspezifische Ziele. Die dialogmusterspezifischen Ziele beziehen sich auf die Inhalte des Beratungsgesprächs, die speziell im Berufs- und Weiterbildungsberatungsprozess verfolgt werden. Dialogmusterunspezifische Ziele beziehen sich 
auf den Kommunikationsprozess im engeren Sinne und die interaktive Gestaltung in der Beratung. Die erarbeiteten Kategorien wurden in einem weiteren Durchgang an die verschiedenen Beratungsfälle durch die Mitglieder der Forscherinnengruppe erneut angelegt. Dann sind diese allein durchgeführten kategorialen Zuordnungen in der Gruppe diskutiert, ausgewertet und in Bezug auf die jeweiligen Beratungsgespräche festgelegt worden. Die dabei erarbeiteten Kategorien betrachten wir als wesentliche Teilschritte im Beratungsprozess, die allerdings keiner Linearität unterliegen. In Anlehnung an Kohl (1986) nennen wir sie ebenfalls dialogmusterspezifische Ziele. Besondere Anforderungen stellte die Identifikation von dialogmusterunspezifischen Zielen dar. Interessanter Weise sind sie im Verständnis von Beratung, selbst in den Beratungstheorien, die wesentlichen Ziele, die intensiv in Beratungsdiskursen besprochen werden. Die dialogmusterspezifischen und dialogmusterunspezifischen Ziele untergliedern sich wiederum in verschiedene kommunikative Teilziele, die zur Beantwortung der Forschungsfrage, welche Verlaufsformen sich in der Berufs- und Weiterbildungsberatung als professionelle Praktiken zur Unterstützung des individuellen Entscheidungsprozesses herausgebildet haben, beitragen. Gleichzeitig verweisen die dialogmusterspezifischen und dialogmusterunspezifischen Ziele auf das primäre Kommunikationsziel: Beratung ist somit eine künstlich erzeugte Kommunikation zum Zwecke von Entscheidungsfindung.

\subsection{Ausdifferenzierung der dialogmusterspezifischen kommunikativen Teilziele}

Aus den vorliegenden Beratungsgesprächen konnten wir elf dialogmusterspezifische kommunikative Teilziele erarbeiten, die keiner Linearität im Beratungsprozess unterliegen und auch nicht in jedem Beratungsgespräch inhaltlich thematisiert werden. Je nach Ausgangssituation und Lebensphase der Ratsuchenden werden die herausgearbeiteten dialogmusterspezifischen kommunikativen Teilziele und ihre Sequenzmustern individuell an die Inhalte des Beratungsgesprächs angepasst.

Folgende dialogmusterspezifische kommunikative Teilziele können benannt werden:

- Abklärung des Beratungsanliegens

- Feststellung der individuellen Berufseinstiegsbedingungen und Berufsverläufe

- Klärung des bisherigen Engagements

- Klärung von Weiterbildungs- und Berufs-(ausbildungs-)vorstellungen, Wünschen und (eigenen) Interessen

- Informationsweitergabe zu den Weiterbildungs-, Berufs-(ausbildungs-) und Studienwünschen, ihrer Bedeutung und ihren Inhalten (Alternativen)

- Suche nach alternativen Weiterbildungs-, Berufs-(ausbildungs-) und Studienmöglichkeiten

- Suche nach Adressen von Weiterbildungs- sowie Arbeitsangeboten und Ausbildungsstätten in verschiedenen institutionellen Kontexten im Wunschgebiet

- Anleitung zum Vorgehen bei der Recherche für eine Weiterbildung, eine Arbeitsstelle, ein Praktikum, eine Ausbildung oder ein Studium

- SichtungundBewertungderBewerbungsunterlagensowieÜberarbeitungshinweise 
- Hinweise für die Weiterbildungs-, Berufs-(ausbildungs-) oder Studium-Suche (Vorgehen)

- Klärung der weiteren Zusammenarbeit

Eingerahmt wird das jeweilige Beratungsgespräch durch die Abklärung des Beratungsanliegens als Einstieg in die Beratung und die Klärung der weiteren Zusammenarbeit als häufiger Ausstieg aus dem Beratungsgespräch. Dazwischen herrscht im Beratungsprozess ein individuelles nicht lineares Zusammenspiel der folgenden kommunikativen Teilziele.

Die Feststellung der individuellen Berufseinstiegsbedingungen und Berufsverläufe unterliegt verschiedenen Themenschwerpunkten (u. a. Schulabschluss, fachspezifische Noten, berufliche Erfahrungen, Berufsausbildung) und bezieht die aktuelle berufliche Situation der Ratsuchenden mit ein. Dort hinein spielt die Klärung des bisherigen Engagements. Welche (beruflichen) Vorerfahrungen können mit Blick auf den Berufswunsch oder die Veränderung der beruflichen Situation eingebracht werden? Daher gilt es auch die Klärung von Weiterbildungs- und Berufs-(ausbildungs-) vorstellung, Wünschen und (eigenen) Interessen vorzunehmen. Beratende und Ratsuchende sondieren gemeinsam das Berufsfeld und erarbeiten individuell anschlussfähige Suchoptionen. Dabei werden die Wünsche, und Interessen der Ratsuchenden thematisiert, um daraus sich ergebende berufliche Anschlussmöglichkeiten zu identifizieren. Diese drei kommunikativen Teilziele halten die Ausgangsbedingungen und die Entscheidungsspielräume der Ratsuchenden fest. Dadurch werden persönliche Ambivalenzen sowie Perspektiven je nach Lebensalter, Biografie und Entscheidungsherausforderungen sichtbar.

Die Informationsweitergabe zu den Weiterbildungs-, Berufs-(ausbildungs-) oder Studienwünschen, ihrer Bedeutung und ihren Inhalten bezieht sich auf die objektiven Voraussetzungen und Möglichkeiten in den Weiterbildungs-, Berufs-, Ausbildungsund Studienfeldern, die von den Beratenden dargelegt werden. Dazu wird konkretes Wissen weitergegeben. Gleichzeitig soll der Blick für die berufliche Entscheidung nicht eingeengt werden, so dass ebenfalls eine Suche nach alternativen Weiterbildungs-, Berufs-(ausbildungs-) oder Studienmöglichkeiten stattfindet. Die erarbeiteten Berufsvorstellungen werden beruflichen Alternativen gegenübergestellt, die ebenfalls die individuellen Wünsche und Interessen der Ratsuchenden im Blick behalten. Letztlich geht es um das Herausfinden von spezifischen Möglichkeitsräumen für den weiteren Entscheidungsverlauf. Die Festlegung auf bestimmte Berufsbereiche ermöglicht die Suche nach Adressen von Weiterbildungs- sowie Arbeitsangeboten und Ausbildungsstätten in verschiedenen institutionellen Kontexten im Wunschgebiet. Diese Suche erfolgt häufig gemeinsam am Computer und wird von den Beratenden begleitet. Bei der Anleitung der Vorgehensweise bei der Recherche für eine Weiterbildung, eine Arbeitsstelle, ein Praktikum, eine Ausbildung oder ein Studium geht es gegenüber der konkreten Suche um Erläuterungen von verschiedenen Recherchemethoden und Zugängen durch die Beratenden zur selbstständigen Ermittlung passender Weiterbildungs-, Arbeits- oder Ausbildungsstellen. Hier wie in den folgenden zwei kommunikativen Teilzielen werden Handlungsschritte und Handlungsziele erarbeitet. Die Sichtung der Bewerbungsunterlagen sowie Überarbeitungshinweise wird allerdings nur in wenigen Beratungsgesprächen aufgegriffen. Bei den Hinwei- 
sen für die Ausbildungs-, Weiterbildungs-, Berufs- oder Studium-Suche geht es konkret darum wie u. a. Vorstellungsgespräche geführt werden könnten.

Die dialogmusterspezifischen kommunikativen Teilziele bewegen sich demnach auf einer thematisch-inhaltlichen Ebene, die sowohl von den Ratsuchenden als auch den Beratenden eingebracht und in der Tendenz gemeinsam bearbeitet wird. Bei der Bearbeitung dieser Inhalte übernehmen die dialogmusterunspezifischen Teilziele eine entscheidende Rolle.

\subsection{Ausdifferenzierung der dialogmusterunspezifischen kommunikativen Teilziele}

Die dialogmusterunspezifischen kommunikativen Teilziele, die wir in unseren Auswertungen der Beratungsgespräche identifizieren konnten, fokussieren zum einen die Aktivitäten zur Beziehungsausgestaltung durch die Beratenden und zum anderen die Aktivitäten zur Erweiterung des kommunikativen Handlungsradius der Ratsuchenden durch die Beratenden. Sie beschreiben damit den dialogischen Prozess selbst und fördern die Herstellung einer vertrauensvollen Atmosphäre. Dabei sind die Sequenzmuster, welche den zwei genannten kommunikativen Teilzielen zugeordnet werden, in ihrer Betrachtung nicht nur induktiv erschlossen, sondern greifen die Ansprüche aus den beratungstheoretischen Diskursen auf. Das bedeutet in diesem Fall auch, dass die professionellen Praktiken sich nach unseren Befunden keinesfalls explizit an einer Theorie orientieren. Wir haben es mit einem eklektizistischen Vorgehen zu tun.

Folgende dialogmusterunspezifische kommunikative Teilziele mit Sequenzmustern als Untergliederung können benannt werden:

- Aktivitäten zur Beziehungsausgestaltung durch die Beratenden

- Verstehen

- Reagieren (Intervenieren, Spiegeln, Interpretieren)

- Bewerten

- Aktivitäten zur Erweiterung des kommunikativen Handlungsradius der Ratsuchenden

- Entwicklung eigener Anfragen, Meinungen und Ideen von den Ratsuchenden aus

- Eröffnung von Erfahrungsräumen durch die Beratenden und Nutzung der Erfahrungsräume durch die Ratsuchenden

- Anregung zur Selbstexploration/Selbstreflexion der Ratsuchenden durch die Beratenden

Das erste kommunikative Teilziel Aktivitäten zur Beziehungsausgestaltung durch die Beratenden nimmt unter dem Sequenzmuster Verstehen beziehungsförderliche Verständnis- und Präzisierungsfragen in den Blick. Durch so angelegte Verstehensprozesse werden zum einen das persönliche Handeln und die persönlichen Interessen der Ratsuchenden nachvollziehbar, zum anderen wird damit die Beziehung zwischen Ratsuchenden und Beratenden entwickelt. Die unter dem Sequenzmuster Reagieren gruppierten Reaktionsformen verdeutlichen bestimmte Beratungstheorien: Das Intervenieren verweist auf systemische Vorgehensweisen, das Spiegeln auf klienten- 
zentrierte Beratung und das Interpretieren auf eher ressourcenorientierte Beratung. Intervenieren zielt darauf ab, die Ratsuchenden herauszufordern, um individuelle Interessen sowie Einstellungen besser herauszuarbeiten und das Nachdenken darüber zu fokussieren. Durch das Spiegeln werden von den Beratenden implizite emotionale Mitteilungen der Ratsuchenden verbalisiert, um das Nachdenken über mögliche Ambivalenzen voranzubringen. Daneben werden durch das Interpretieren Vermutungen zum Gesagten durch die Beratenden geäußert, um inhaltliche Zwischenergebnisse festzuhalten. Bewerten als Sequenzmuster schließt direkte und indirekte Meinungsäußerungen der Beratenden ein.

Das zweite kommunikative Teilziel fokussiert Aktivitäten der Beratenden, welche die kommunikativen Selbstaktivitäten der Ratsuchenden unterstützen. Dabei wird im Sequenzmuster Entwicklung eigener Anfragen, Meinungen und Ideen von den Ratsuchenden aus deren eingebrachter Kommunikationsradius dokumentiert. Hierbei handelt es sich um das einzige Sequenzmuster, welches in der Beziehungsausgestaltung die Perspektive der Ratsuchenden einnimmt. Es ist uns wichtig, diesen Aspekt mit aufzugreifen, da in der Regel die jungen Erwachsenen die von uns erwartete verbale Rolle im Beratungsgespräch nicht einnehmen. Für Erwachsene gilt das nicht. Die Eröffnung von Erfahrungsräumen durch die Beratenden nimmt Denkanstöße, die durch die Beratenden gegeben werden, in den Blick und dokumentiert diese. Dabei wird diese Kategorie durch die Ratsuchenden keineswegs so genutzt, wie unterstellt. Die Nutzung der Erfahrungsräume durch die Ratsuchenden ist somit nicht zwingend gegeben. Zum einen liegt das daran, dass der kommunikative Raum durch die Beratenden nicht gesetzt wird, zum anderen, dass die Ratsuchenden auf die gegebenen Denkanstöße nicht eingehen oder sie nicht erkennen. Als ein weiteres Sequenzmuster unter diesem kommunikativen Teilziel wird von den Beratenden die Anregung zur Selbstexploration/Selbstreflexion der Ratsuchenden initiiert. Auch dieses Sequenzmuster wird häufig nicht im Beratungsgespräch ausgehandelt, das heißt die Anregungen werden von den Ratsuchenden nicht direkt genutzt. Die Erfahrungsräume der Ratsuchenden, die durch die Beratenden bewusst durch Denkanstöße und Anregungen zur Selbstexploration offen gehalten werden, korrespondieren somit nicht mit einer entsprechenden Nutzung durch die Ratsuchenden.

Durch die Analyse der Beratungsgespräche mit Blick auf die dialogmusterunspezifischen Ziele kann festgestellt werden, dass - wenn es sich um einen dialogischen Prozess handeln soll - für die Ratsuchenden noch mehr Kommunikationsraum vorhanden sein müsste, da diese sich sonst verstärkt in der Rolle des Bestätigens, Verneinens oder nur Zuhörens begeben.

In der abgeschlossenen Untersuchung dokumentieren wir an exemplarischen Beispielen wie dialogmusterspezifische und dialogmusterunspezifische Ziele in hoher Komplexität im Beratungsverlauf zusammenwirken (Gieseke et al. 2014; Gieseke und Stimm 2015, i. B.).

\section{Zusammenfassende Erkenntnisse}

Die von uns herausgearbeiteten dialogmusterspezifischen und dialogmusterunspezifischen Ziele möchten wir nicht im Sinne eines Modells für Beratungsprozesse 
verstanden wissen, sondern als Verweis auf verschiedene empirisch gewonnene Kategorien in den professionellen Beratungspraktiken.

Die erschlossenen dialogmusterspezifischen Ziele, die auf der inhaltlich-thematischen Ebene in der Beratung verfolgt werden, verweisen auf die komplexe Wissensanforderung an die Beratenden (auch Enoch 2011). Nicht umsonst haben wir es mit einer Dominanz der dialogmusterspezifischen Ziele zu tun. Hinsichtlich der dialogmusterunspezifischen Ziele, die auf die Beziehungs- und Kommunikationsebene im Beratungsprozess verweisen, muss weitergeforscht werden. Nur so viel lässt sich aufgrund der vorliegenden Ergebnisse schon festhalten - auch bedingt durch andere Befunde (Müller 2005; Schreyögg 2015): Jeder geäußerte Satz in der Beratung hat eine Bedeutung und bleibt deshalb nicht ohne Wirkung, wobei auch die Haltung des Individuums in der Situation indirekt mit kommuniziert wird.

Unsere Untersuchung, die in diesem Jahr veröffentlicht wird (Gieseke und Stimm 2015, i. B.), zeigt, wie komplex - angelegt in der Praxis - Beratung geschieht und auf welchen Ebenen Kompetenzen in jeder Beratungssituation gefragt sind. Die hohe Bedeutung von systematischem, theoretischem und empirischem Wissen zu den Beratungsgegenständen, also in unserem Fall von Berufs- und Weiterbildungsstrukturen, kann nicht mehr übersehen werden. Ebenso ist ein Wissen über Sozialisationsprozesse und soziale, auch milieuspezifisch individuelle Verhaltensmuster notwendig. Eine Grundvoraussetzung ist ein reflexiv bearbeitetes Menschenbild auf ethischer Grundlage. Hinzu kommen dispositive Fähigkeiten wie große Offenheit, Authentizität und schnelles Einordnen der Situation und Anforderungen sowie Reagieren. Innere Selbstdisziplin und reflexive Kompetenz, mit den eigenen Projektionen umzugehen, sind ebenfalls notwendig. Die fehlende Beachtung dieser genannten Aspekte hat nicht nur Folgen für die jeweiligen Ratsuchenden, sondern sie hat auch gesellschaftliche Bedeutung, da von den Ratsuchenden ansonsten zu viele Umwege eingeschlagen werden und Individuen letztlich nicht selbstständig entscheidungsfähig werden.

Open Access Dieser Artikel wird unter der Creative Commons Namensnennung 4.0 International Lizenz (http://creativecommons.org/licenses/by/4.0/deed.de) veröffentlicht, welche die uneingeschränkte Nutzung, Verbreitung und Wiedergabe für beliebige Zwecke erlaubt, sofern Sie den/die ursprünglichen Autor(en) und die Quelle ordnungsgemäß nennen, einen Link zur Creative Commons Lizenz beifügen und angeben, ob Änderungen vorgenommen wurden.

\section{Literatur}

Agentur für Erwachsenen- und Weiterbildung. (Hrsg.). (2013). Bildungsberatung - Orientierung, Offenheit, Qualität. Die niedersächsischen Modellprojekte (Aktuelles aus der Erwachsenen- und Weiterbildung, Bd. 2). Bielefeld: W. Bertelsmann Verlag.

Arnold, R., Gieseke, W., \& Zeuner, C. (Hrsg.). (2009). Bildungsberatung im Dialog. Band I: Theorie - Empirie - Reflexion. Band II: 13 Wortmeldungen. Band III: Referenzmodelle. Baltmannsweiler: Schneider Verlag.

Bohnsack, R. (2008). Rekonstruktive Sozialforschung. Einführung in qualitative Methoden. Opladen \& Farmington Hills: Verlag Barbara Budrich.

de Cuvry, A., Kossack, P., \& Zeuner, C. (2009). Strukturmodell für personenbezogene Bildungsberatung. In R. Arnold, W. Gieseke, \& C. Zeuner (Hrsg.), Bildungsberatung im Dialog. Band III: Referenzmodelle (S. 135-159). Hohengeheren: Schneider Verlag. 
Damasio, A. R. (2000). Descartes' Irrtum. Fühlen, Denken und das menschliche Gehirn. München: List Verlag.

Damasio, A. R. (2003). Der Spinoza-Effekt. Wie Gefühle unser Leben bestimmen. München: List.

Deppermann, A., Reitmeier, U., Schmitt, R., \& Spranz-Fogasy, T. (Hrsg.). (2010). Verstehen in professionellen Handlungsfeldern. Tübingen: Narr Francke Attempto Verlag.

Enoch, C. (2011). Die Wissensdimension in allgemeinen Beratungsansätzen. Wiesbaden: VS Verlag für Sozialwissenschaften.

Ertelt, B.-J., \& Schulz, W. E. (2002). Handbuch Beratungskompetenz. Mit Übungen zur Entwicklung von Beratungsfertigkeiten in Bildung und Beruf. Unter Mitarbeit von Allen E. Ivey. Leonberg: Rosenberger Fachverlag.

Fuchs, T. (2008). Leib und Lebenswelt. Neue philosophisch-psychiatrische Essays. Kusterdingen: Die Graue Edition.

Fuchs, T. (2010). Das Gehirn - ein Beziehungsorgan. Eine phänomenologisch-ökologische Konzeption. Stuttgart: W. Kohlhammer Verlag.

Fuhr, R. (2003). Struktur und Dynamik der Berater-Klient-Beziehung. In C. Krause, B. Fittkau, R. Fuhr, \& H.-U. Thiel (Hrsg.), Pädagogische Beratung. Grundlagen und Praxisanwendung (S. 32-50). Paderborn: Verlag Ferdinand Schöningh.

Gieseke, W. (2009). Lebenslanges Lernen und Emotionen. Wirkungen von Emotionen auf Bildungsprozesse aus beziehungstheoretischer Perspektive. Bielefeld: W. Bertelsmann Verlag.

Gieseke, W. (2013). Orientierungsberatung. In Agentur für Erwachsenen- und Weiterbildung (Hrsg.), Bildungsberatung - Orientierung, Offenheit, Qualität. Die niedersächsischen Modellprojekte (S. 15-35). (Aktuelles aus der Erwachsenen- und Weiterbildung, Bd. 2). Bielefeld: W. Bertelsmann Verlag.

Gieseke, W. (2014a). Studienberatung zwischen Bildungsberatung, Lernberatung und psychologischer Beratung. Zeitschrift für Beratung und Studium. Handlungsfelder, Praxisbeispiele und Lösungskonzepte 1, 10-14.

Gieseke, W. (2014b). Entscheidungsfähigkeit des Individuums als Bildungsberatungsherausforderung die Wahl haben? In M. Ebner von Eschenbach, S. Günther, \& A. Hauser (Hrsg.), Gesellschaftliches Subjekt. Erwachsenenpädagogische Perspektiven und Zugänge (S. 206-219). Hohengehren: Schneider Verlag.

Gieseke, W., \& Stimm, M. (2015, i. B.). Bildungsberatungspraktiken - Grundlagen für die Entscheidungsfindung von Subjekten. Wiesbaden: Springer VS.

Gieseke, W., Dietel, S., \& Ebner von Eschenbach, M. (2012). Referenzmodell für die Koordination bildungsbereichs- und trägerübergreifender Bildungsberatung im Rahmen eines Bildungsmanagements auf kommunaler Ebene. Projektbericht im Rahmen der 1. Förderphase des Programms „Lernen vor Ort". unv.

Gieseke, W., Stimm, M., \& Putensen, M. (2014). Dialogmusteranalyse am Beispiel der Studienberatung. In A. Schlüter (Hrsg.), Beratungsfälle. Fallanalysen für die Lern- und Bildungsberatung (S. 79-102). Opladen: Barbara Budrich.

Gigerenzer, G. (2007). Bauchentscheidungen. Die Intelligenz des Unbewussten und die Macht der Intuition. München: C. Bertelsmann Verlag.

Göckler, R. (2012). Beschäftigungsorientiertes Fallmanagement. Betreuung und Vermittlung in der Grundsicherung für Arbeitsuchende (SGB II). Case Management in der Praxis. Regensburg: Walhalla Fachverlag.

Käpplinger, B., Klein, R., \& Haberzeth, E. (Hrsg.). (2013). Weiterbildungsgutscheine - Wirkungen eines Finanzierungsmodells in vier europäischen Ländern. Bielefeld: W. Bertelsmann Verlag.

Kohl, M. (1986). Zielstrukturen und Handlungsorganisation im Rahmen von Dialogmusterbeschreibungen. In F. Hundsnurscher \& E. Weigand (Hrsg.), Dialoganalyse (S. 51-67). Tübingen: Niemeyer.

Kruse, W., Kaletka, C., Pelka, B., \& Schröder, A. (2009). Hessencampus 2007-2009. Veröffentlichung der wissenschaftlichen Begleitung. (Beiträge aus der Forschung, Bd. 173). http://www.sfs.tu-dortmund. $\mathrm{de} / \mathrm{odb} /$ Repository/Publication/Doc/1228/badf_band_173.pdf.

Lippitt, G., \& Lippitt, R. (1999). Beratung als Prozess. Was Berater und ihre Kunden wissen - sollten. Leonberg: Rosenbergerfachverlag.

Maier-Gutheil, C. (2009). Zwischen Beratung und Begutachtung. Pädagogische Professionalität in der Existenzgründungsberatung. Wiesbaden: VS Research.

Müller, A. (2005). Weiterbildungsberatung: Qualitative Analyse von Interaktions- und Prozessverläufen situativer und biographieorientierter Weiterbildungsberatungsgespräche. Berlin: Rhombos-Verlag. 
Perler, D. (2011). Transformationen der Gefühle. Philosophische Emotionstheorien 1270-1670. Frankfurt am Main: S. Fischer Verlag.

Reckwitz, A. (2000). Die Transformation der Kulturtheorien. Zur Entwicklung eines Theorieprogramms. Weilerswist: Velbrück.

Reckwitz, A. (2003). Grundelemente einer Theorie sozialer Praktiken: Eine sozialtheoretische Perspektive. Zeitschrift für Soziologie, 4, 282-301.

Roth, G. (2001). Fühlen, Denken, Handeln. Wie das Gehirn unser Verhalten steuert. Frankfurt am Main: Suhrkamp Verlag.

Roth, G. (2008). Persönlichkeit, Entscheidung und Verhalten: Warum es so schwierig ist, sich und andere zu ändern. Stuttgart: Klett-Cotta.

Schemmann, M., \& Seitter, W. (2013). Bildungsberatungsbericht Hessencampus 2013. http://www.vhs-inhessen.de/Artikel/cmx53ce320d1bc0c.html.

Schiersmann, C., \& Remmele, H. (2004). Beratungsfelder in der Weiterbildung: Eine empirische Bestandsaufnahme. Hohengehren: Schneider Verlag.

Schiersmann, C., \& Thiel, H.-U. (Hrsg.). (2012). Beratung als Förderung von Selbstorganisationsprozessen. Empirische Studien zur Beratung von Personen und Organisationen auf der Basis der Synergetik. Göttingen: Vandenhoeck \& Ruprecht.

Schiersmann, C., \& Weber, P. (Hrsg.). (2013). Beratung in Bildung, Beruf und Beschäftigung. Eckpunkte und Erprobung eines integrierten Qualitätskonzepts. Bielefeld: W. Bertelsmann Verlag.

Schreyögg, B. (2015). Emotionen im Coaching. Kommunikative Muster der Beratungsinteraktion. Wiesbaden: Springer Fachmedien.

Schröder, F., \& Schlögl, P. (2014). Weiterbildungsberatung. Qualität definieren, gestalten, reflektieren. Bielefeld: W. Bertelsmann Verlag.

Thiel, H.-U. (2003). Phasen des Beratungsprozesses. In C. Krause, B. Fittkau, R. Fuhr, \& H.-U. Thiel (Hrsg.), Pädagogische Beratung. Grundlagen und Praxisanwendung (S. 73-84). Paderborn: Verlag Ferdinand Schöningh.

Tippelt, R., \& Barz, H. (Hrsg.). (2007). Weiterbildung und soziale Milieus in Deutschland. Praxishandbuch Milieumarketing. Bielefeld: W. Bertelsmann Verlag.

Wrana, D. (2015). Subjekt. In J. Dinkelaker \& A. von Hippel (Hrsg.), Erwachsenenbildung in Grundbegriffen (S. 34-41). Stuttgart: Kohlhammer. 